\title{
Importance of genetics in acute myeloid leukemia
}

\section{Papel de la genética en la leucemia mieloide aguda}

\author{
R. Pippa, M.D. Odero
}

\begin{abstract}
Acute myeloid leukemia (AML) comprises a biologically and clinically heterogeneous group of aggressive disorders that occur as a consequence of a wide variety of genetic and epigenetic abnormalities in hematopoietic progenitors. Despite significant advances in the understanding of the biology of AML, most patients will die from relapsed disease. Whole-genome studies have identified novel recurrent gene mutations with prognostic impact in AML; furthermore, it is likely that in the near future genome-wide sequencing will become a routine for newly diagnosed patients with AML. Therefore, future clinical trials should aim to identify genetically defined high-risk patients, and further research is necessary to identify effective agents and develop new individualized therapeutic strategies for the treatment of this deadly disease.
\end{abstract}

Key words. Acute myeloid leukemia. Genetic marker. Mutation. Whole-genome sequencing. Prognosis.

\section{RESUMEN}

La leucemia mieloide aguda (LMA) es una enfermedad clínica y molecularmente heterogénea, que surge como consecuencia de alteraciones genéticas y epigenéticas adquiridas en células progenitoras hematopoyéticas. A pesar de los avances realizados en el conocimiento de la biología de esta enfermedad, la supervivencia global de los pacientes sigue siendo muy baja debido principalmente a la alta tasa de recaídas. Los estudios de secuenciación masiva han permitido la identificación de nuevas mutaciones recurrentes y con impacto pronóstico en LMA; además, parece probable que en poco tiempo la secuenciación de todo el genoma sea una prueba diagnóstica de rutina, lo que permitirá el diagnóstico molecular de los pacientes. Por tanto, es necesario el desarrollo de dianas moleculares que abran nuevas perspectivas terapéuticas y permitan el tratamiento individualizado de los pacientes con esta enfermedad tan agresiva.

Palabras clave. Leucemia mieloide aguda. Marcador genético. Mutación. Secuenciación masiva. Pronóstico.
Department of Biochemistry and Genetics and CIMA. University of Navarra.

This study was supported by Ministerio de Ciencia e Innovación (PI11/02443), Departamento Salud del Gobierno de Navarra (78/2012), ISCIIIRTICC (RD12/0036/0063) and Fundación para la Investigación Médica Aplicada (Spain).

\section{Correspondencia:}

M.D. Odero

Department of Biochemistry and Genetics and CIMA University of Navarra

C/ Pío XII, 55

31008-Pamplona, Spain.

E-mail: modero@unav.es

Recepción: 1 de noviembre de 2013

Aceptación provisional: 26 de mayo de 2014

Aceptación definitiva: 29 de septiembre de 2014 


\section{INTRODUCTION}

Acute myeloid leukemia (AML) comprises a biologically and clinically heterogeneous group of aggressive disorders that occur as a consequence of a wide variety of genetic and epigenetic abnormalities in hematopoietic progenitors. Despite significant advances in the understanding of the biology of AML, overall survival remains poor due chiefly to the high rate of relapse after achieving complete remission, as well as primary failure of induction chemotherapy ${ }^{1}$.

Since it is predominantly a disease of older people, the therapeutic strategy offered for AML is determined by assessment of the patient's age and general fitness level. The standard of care for over 3 decades has been the combination of daunorubicin with cytarabine (Ara-C). The majority of younger patients achieve a complete remission (CR) (75\%), and 40\%-45\% will have a chance of cure. This represents a steady improvement over the past 40 years, although much of this can be attributed to improvements in supportive care, much of which has been learned from the transplantation experience ${ }^{2}$. A major challenge is the treatment of older patients, defined arbitrarily as over 60 years, who represent the majority of patients with this disease. Approximately $50 \%$ of older patients reach CR, but $85 \%$ of those entering remission will relapse, and the outcome from relapse in older patients is poor, with relatively few surviving. In addition, there are a substantial number of patients who are not considered suitable for intensive chemotherapy, for whom a palliative approach is usually offered $^{2}$.

Continuing research into the pathogenesis and heterogeneity of AML has resulted in the development of several potentially useful therapeutic agents. Notably, acute promyelocytic leukemia (APL) has much better prognosis due to the implementation of sensitive molecular diagnostic tools, and to the introduction of all-trans retinoic acid (ATRA) in combination with anthracycline into clinical practice. Clinical trials with patients with APL have reported complete remission and long-term disease-free survival rates of approximately $90 \%$ and $85 \%$, respectively ${ }^{3}$. Other therapies targeting specific molecular defects are being developed, such as small molecule inhibitors of FLT3 kinase in patients harboring the FLT3ITD mutation, and all-trans retinoic acid in patients with the NPM1 mutation. However, despite some advances in the treatment of AML, the overall outcome is still dismal for most patients.

\section{CYTOGENETIC AND MOLECULAR ABERRATIONS WITH PROGNOSTIC RELEVANCE IN AML}

Several studies over the past decades have identified a large set of chromosome aberrations, mutations and overexpressed genes with prognostic relevance in AML, improving our understanding of AML pathogenesis and risk stratification. AML patients can be classified into three different prognostic subgroups according to presence or absence of distinct cytogenetic abnormalities. Moreover, in recent years, various molecular genetic markers have been identified, especially in normal karyotype (NK) AML, where screening for mutations in genes such as FLT3, NPM1, and CEBPA has allowed better prognostic prediction (Table 1$)^{4,5}$. At present, most patients can be categorized into clinicopathological subgroups based on the presence of genetic defects. Our increasing knowledge of AML biology led to the establishment of the 2008 World Health Organization classification, which considers morphologic features and recurrent cytogenetic and molecular abnormalities ${ }^{6}$. In addition, an international expert panel from the European LeukemiaNet (ELN) has also recently proposed new guidelines for the management and stratification of therapies based on the strongest prognostic factors identified to date such as cytogenetic or molecular defects?.

However, a large number of AML patients lack any of these abnormalities and there remains significant heterogeneity in clinical outcome within currently classified 
prognostic groups. These observations suggested that there may be additional biomarkers that can predict outcome in AML. In this regard, recent genome-wide studies have identified an increasing number of recurrent somatic mutations in AML patients, such as mutations in ASXL1, DNMT3A, IDH1, IDH2, PHF6 and TET2 ${ }^{4}$. Several of these newly identified genetic abnormalities have prognostic importance in AML. Moreover, the identification of mutations at diagnosis has served as a tool for minimal residual disease measurement. Interestingly, a recent study has provided a large-scale insight into the genetics of relapsed AML by performing whole-genome sequencing of 8 patients with relapsed $\mathrm{AML}^{8}$. In all patients there was a founding clone that was not ablated by chemotherapy and was still persistent at relapse; thus, prospective identification of this clone could be of great clinical utility.

\section{MOLECULAR MARKERS IN NORMAL KARYOTYPE ACUTE MYELOID LEUKEMIA}

Mutational and wide-genomic studies have been especially useful to increasingly refine prognosis in patients with intermediate-risk and/or NK-AML, who represent more than $45 \%$ of all AML cases. The 5-year survival rate of these patients varies from $24 \%$ to $42 \%$, suggesting a genetic diversity within this subgroup. As indicated above, previous studies have shown that mutational analysis of FLT3, NPM1, and CEBPA can be used to risk-stratify these patients ${ }^{5}$. However, more extensive mutational analyses indicate that other genes should be included in the screening, and that mutations analysis of at least ten genes: ASXL1, CEBPA, DNMT3A, FLT3, IDH1, IDH2, MLL, NPM1, PHF6, and TET2, would represent a useful tool, discriminating intermediate-risk AML patients into robust, clinically relevant risk groups (Table 1$)^{4}$.

About $20 \%$ to $25 \%$ of AML patients harbor internal tandem duplication (ITD) mutations in the RTK Fms-like tyrosine kinase-3 (FLT3), which leads to constitutive phosphorylation of FLT3 and activation of its downstream signaling cascades, resulting ultimately in aberrant proliferation and survival. FLT3-ITD mutations have been consistently found to be an adverse prognostic marker in NK-AML patients, associated with an increased risk of relapse and inferior overall survival ${ }^{9}$. This has led to clinical trials treating FLT3-ITD AML patients with small-molecule FLT3-selective tyrosine kinase inhibitors. To date, however, FLT3 inhibitors used as a single agent or in combination with chemotherapy for FLT3-ITD AML have not demonstrated improved clinical efficacy ${ }^{10}$. Because FLT3 can crosstalk with a network of various signaling pathways, identifying and analyzing the interplay of constitutively active FLT3 with aberrant signaling pathways may lead to the identification of novel therapeutic targets for treatment of AML patients harboring constitutively active FLT $3^{11}$. Several studies have shown that risk associated with FLT3-ITD in AML may depend on mutational burden and its interaction with other mutations ${ }^{12,13}$. In the subset of patients with FLT3-ITD-negative intermediate-risk AML, there are 3 distinct risk groups that are based on mutational status and have vastly different outcomes. FLT3-ITD-negative, NPM1/IDH mutant patients have outcomes that are better than patients with inv(16)or $t(8: 21)$-positive AML, suggesting that this represents a favorable-risk AML subset defined by a specific mutational genotype. In contrast, FLT3-ITD-negative NPM1 mutant patients without concurrent IDH mutations have a much less favorable outcome. Most importantly, the presence of poor-risk mutations, specifically TET2, ASXL1, PHF6, and/or MLL-PTD, is associated with very adverse overall survival for FLT3-ITD wildtype, intermediate-risk patients ${ }^{14}$. These data suggest that NPM1 mutational status alone does not define a favorable subset of intermediate-risk AML, and that the presence or absence of additional disease alleles defines relapse risk in FLT3-ITD wildtype, intermediate-risk AML ${ }^{14}$. In addition, mutational studies have allowed for improved prognostication in FLT3-ITD-positive patients (Table 1$)^{4,14}$. These results 
have important clinical implications, because patients with mutationally defined favorable risk have a better outcome with standard induction and consolidation than even patients with core binding factor-positive AML. In contrast, patients with muta- tionally defined adverse-risk AML have an outcome similar to patients with adverse karyotypic risk, and standard therapies are not sufficient to offer curative intent to the majority of these patients $s^{4,14}$.

Table 1. Relevant molecular markers in normal karyotype or intermediate-risk cytogenetic AML (adapted from Patel et al., 2012 ${ }^{14}$ )

\begin{tabular}{l} 
Overall Risk Profile \\
\hline FAVORABLE \\
\hline FLT3-ITD negative \\
Mutant NPM1 and IDH1 or IDH2 \\
\hline INTERMEDIATE \\
\hline FLT3-ITD negative \\
Wild-type ASXL1, MLL-PTD, PHF6 and TET2 \\
FLT3-ITD negative or positive \\
Mutant CEBPA \\
FLT3-ITD positive \\
Wild-type MLL-PTD, TET2 and DNMT3A and trisomy 8 negative \\
\hline UNFAVORABLE \\
\hline FLT3-ITD negative \\
Mutant TET2, MLL-PTD, ASXL1 or PHF6 \\
FLT3-ITD positive \\
Mutant TET2, MLL-PTD, DNMT3A, or trisomy 8, without mutant CEBPA
\end{tabular}

\section{CLINICAL IMPACT OF GENETIC} ABERRATIONS IN ACUTE MYELOID LEUKEMIA

With the discovery of novel genes associated with AML pathogenesis continuing at a high speed, the challenge is to integrate this knowledge into the current clinical understanding of $\mathrm{AML}^{4}$. Nevertheless, in the clinical setting, most AML centers use cytogenetic abnormalities and a relatively small set of gene-based tests to assign risk in AML and to determine post-remission therapy. As Patel and Levine indicate, the relative paucity of clinically used biomarkers is due to several factors. First, most biomarker studies focus on a specific genetic lesion and its prognostic relevance without considering the complete set of known mu- tations in parallel to determine which mutations predict outcome independently in AML. Second, most studies consider each mutant allele as a distinct variable without considering complex genotypes in which the presence/absence of multiple disease alleles has different effects on outcome than individual mutations by themselves. Third, many studies have focused on mutational "hotspots" or have used less sensitive techniques to identify loss-of-function mutations in large tumor suppressors. Finally and most importantly, until recently, most studies of AML were relatively small in size and/or were not derived from clinical trial cohorts in which the effects of treatment on outcome can be controlled and investigated. Thus, given the increasing number of genetic abnormalities that 
have been identified in AML patients, it has become important to determine the prognostic relevance of all known recurrent genetic abnormalities in a uniformly treated AML patient cohort ${ }^{4}$.

\section{CONCLUSIONS AND FUTURE PERSPECTIVES}

Recent advances in the research of AML, especially the identification of novel genetic mutations, have enabled us to stratify this heterogeneous disease entity into distinct subtypes beyond the scopes of cytomorphology and cytogenetics. However, to date, most studies have failed to identify robust predictors that modify outcome in patients with favorable or unfavorable cytogenetic risk, suggesting that chromosomal lesions remain the best predictor of outcome for the $40 \%$ of AML patients with favorable or unfavorable karyotype ${ }^{4}$. Thus, there is a common trend to characterize better AML subtypes as soon as the diagnosis is made. This is exemplified by the subgroup of patients with the monosomal karyotype, who have a dismal outcome with standard treatment, including transplantation ${ }^{15}$. However, in the group of intermediate AML the identification of mutations with impact on outcome has clinical and biological importance, and molecular screenings help to refine the treatment strategies. Therefore, it is important to determine specific mutations or combinations of mutations in this subgroup of patients, although the main goal of future mutational studies should be to inform and improve prognostic algorithms in AML. Moreover, validation of these findings in other large, homogeneously treated patient cohorts is of utmost clinical importance 4 .

As discussed above, application of whole-genome sequencing to AML has already yielded important discoveries, including the identification of common gene mutations with prognostic impact in AML. In the near future, it is likely that whole genome sequencing will become a routine part of the diagnostic workup of patients with $\mathrm{AML}^{16}$. Therefore, future clinical trials should aim to identify genetically defined high-risk patients to offer them novel therapies early in their disease course, in an effort to reduce relapse and increase cure ${ }^{4}$.

Finally, although hundreds of different genetic lesions have been described in AML, this disease shares common programs of self-renewal and transformation downstream of leukemia-associated oncogenes ${ }^{17}$. These findings argue for the presence of common mechanisms of leukemia cell survival, and suggest that mechanistically common therapeutic approaches to AML are likely to be possible ${ }^{18}$. Further research is thus necessary to identify effective agents and develop new individualized therapeutic strategies for the treatment of this deadly disease.

\section{BIBLIOGRAPHY}

1. Ravandi F, Cortes J, Faderl S, O’Brien S, GarCiA-Manero G, VerstovseK $S$ et al. Characteristics and outcome of patients with acute myeloid leukemia refractory to 1 cycle of highdose cytarabine-based induction chemotherapy. Blood 2010; 116: 5818-5823.

2. BuRnETT AK. Treatment of acute myeloid leukemia: are we making progress? Hematology Am Soc Hematol Educ Program 2012; 1-6.

3. Powell Bl, Moser B, Stock W, Gallagher RE, Willman CL, Stone RM et al. Arsenic trioxide improves event-free and overall survival for adults with acute promyelocytic leukemia: North American Leukemia Intergroup Study C9710. Blood 2010; 116: 3751-3757.

4. Patel JP, Levine RL. How do novel molecular genetic markers influence treatment decisions in acute myeloid leukemia? Hematology Am Soc Hematol Educ Program 2012; 2834 .

5. Schlenk RF, Dohner K, Krauter J, Frohling S, Corbacioglu A, Bullinger L et al. Mutations and treatment outcome in cytogenetically normal acute myeloid leukemia. $\mathrm{N}$ Engl J Med 2008; 358: 1909-1918.

6. Vardiman JW, Thiele J, Arber DA, Brunning RD, Borowitz MJ, Porwit A et al. The 2008 revision of the World Health Organization (WHO) classification of myeloid neoplasms and acute leukemia: rationale and important changes. Blood 2009; 114: 937-951.

7. Dohner H, Estey EH, Amadori S, Appelbaum FR, BuCHNER T, Burnett AK et al. Diagnosis and 
management of acute myeloid leukemia in adults: recommendations from an international expert panel, on behalf of the European LeukemiaNet. Blood 2010; 115: 453-474.

8. Ding L, Ley TJ, Larson DE, Miller CA, Koboldt DC, Welch JS et al. Clonal evolution in relapsed acute myeloid leukaemia revealed by whole-genome sequencing. Nature 2012; 481: 506-510.

9. StiREwalt DL, Radich JP. The role of FLT3 in haematopoietic malignancies. Nat Rev Cancer 2003; 3: 650-665.

10. Weisberg E, Barrett R, Liu Q, Stone R, Gray N, GRIFFIN JD. FLT3 inhibition and mechanisms of drug resistance in mutant FLT3-positive AML. Drug Resist Updat 2009; 12: 81-89.

11. Park IK, Mishra A, Chandler J, Whitman SP, MarCUCCI G, CALIGIURI MA. Inhibition of the receptor tyrosine kinase Axl impedes activation of the FLT3 internal tandem duplication in human acute myeloid leukemia: implications for Axl as a potential therapeutic target. Blood 2013; 121: 2064-2073.

12. Gale RE, Green C, Allen C, Mead AJ, Burnett AK, HILLS RK et al. The impact of FLT3 internal tandem duplication mutant level, number, size, and interaction with NPM1 mutations in a large cohort of young adult patients with acute myeloid leukemia. Blood 2008; 111: 2776-2784.
13. Pratcorona M, Brunet S, Nomdedeu J, Ribera JM, Tormo M, Duarte R et al. Favorable outcome of patients with acute myeloid leukemia harboring a low-allelic burden FLT3-ITD mutation and concomitant NPM1 mutation: relevance to post-remission therapy. Blood 2013; 121: 2734-2738.

14. Patel JP, Gonen M, Figueroa ME, Fernandez H, Sun Z, Racevskis J, et al. Prognostic relevance of integrated genetic profiling in acute myeloid leukemia. N Engl J Med 2012; 366: 10791089.

15. Estey E. High cytogenetic or molecular genetic risk acute myeloid leukemia. Hematology Am Soc Hematol Educ Program 2010; 474480.

16. WeLCh JS, LinK DC. Genomics of AML: clinical applications of next-generation sequencing. Hematology Am Soc Hematol Educ Program $2011 ; 30-35$.

17. Cancer Genome Atlas Research Network. Genomic and Epigenomic Landscapes of Adult De Novo Acute Myeloid Leukemia. N Engl J Med 2013; 368: 2059-2074.

18. Kvinlaug BT, Chan WI, Bullinger L, Ramaswami M, SEArs C, Foster D et al. Common and overlapping oncogenic pathways contribute to the evolution of acute myeloid leukemias. Cancer Res 2011; 71: 4117-4129. 